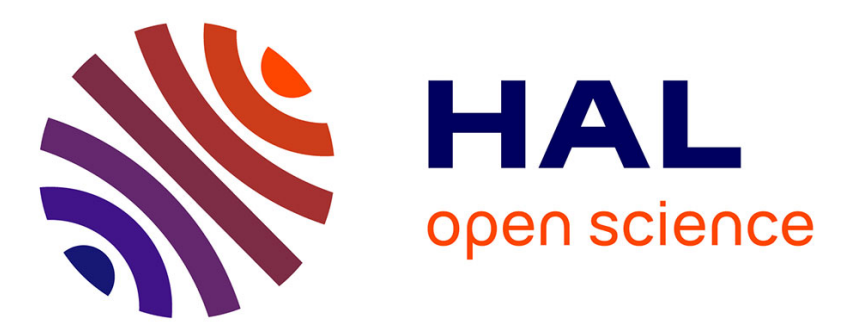

\title{
Dynamics Consensus between Centroidal and Whole-Body Models for Locomotion of Legged Robots
}

Rohan Budhiraja, Justin Carpentier, Nicolas Mansard

\section{To cite this version:}

Rohan Budhiraja, Justin Carpentier, Nicolas Mansard. Dynamics Consensus between Centroidal and Whole-Body Models for Locomotion of Legged Robots. ICRA 2019 - IEEE International Conference on Robotics and Automation, May 2019, Montreal, Canada. hal-01875031v3

\section{HAL Id: hal-01875031 \\ https://hal.laas.fr/hal-01875031v3}

Submitted on 2 Apr 2019

HAL is a multi-disciplinary open access archive for the deposit and dissemination of scientific research documents, whether they are published or not. The documents may come from teaching and research institutions in France or abroad, or from public or private research centers.
L'archive ouverte pluridisciplinaire HAL, est destinée au dépôt et à la diffusion de documents scientifiques de niveau recherche, publiés ou non, émanant des établissements d'enseignement et de recherche français ou étrangers, des laboratoires publics ou privés. 


\title{
Dynamics Consensus between Centroidal and Whole-Body Models for Locomotion of Legged Robots
}

\author{
Rohan Budhiraja ${ }^{a, *}$, Justin Carpentier ${ }^{\text {a,b,c }}$ and Nicolas Mansard ${ }^{a}$
}

\begin{abstract}
It is nowadays well-established that locomotion can be written as a large and complex optimal control problem. Yet, current knowledge in numerical solver fails to directly solve it. A common approach is to cut the dimensionality by relying on reduced models (inverted pendulum, capture points, centroidal). However it is difficult both to account for whole-body constraints at the reduced level and also to define what is an acceptable trade-off at the whole-body level between tracking the reduced solution or searching for a new one. The main contribution of this paper is to introduce a rigorous mathematical framework based on the Alternating Direction Method of Multipliers, to enforce the consensus between the centroidal state dynamics at reduced and whole-body level. We propose an exact splitting of the whole-body optimal control problem between the centroidal dynamics (under-actuation) and the manipulator dynamics (full actuation), corresponding to a re-arrangement of the equations already stated in previous works. We then describe with details how alternating descent is a good solution to implement an effective locomotion solver. We validate this approach in simulation with walking experiments on the HRP-2 robot.
\end{abstract}

\section{INTRODUCTION}

\section{A. Motivation}

Trajectory optimization for generating dynamically feasible motions remains a complex and challenging problem for legged robots. The main difficulty arises from the non-convex dynamics with numerous Degrees of Freedom (DoF) which must be solved together to create a feasible and optimal solution.

Much of the recent efforts in the community have been focused on reducing the dimensionality and the complexity of the problem by relying on reduced models (e.g. table-cart [1], capture point [2] etc). One such approach which has recently gained in popularity is based on computing first the reference trajectory for the centroidal dynamics [3] of the robot, and then using this trajectory to generate a whole-body motion which is dynamically consistent [4], [5], [6]. It is easy to understand the reason behind this popularity: the problem is divided into two consecutive subproblems of smaller dimensions than the original problem, and thus are individually easier to solve. In addition, and contrary to other approaches, centroidal dynamics is exact projection of the full dynamics, which

This work is supported by the FLAG-ERA JTC 2016 RobCom++ project, the European project MEMMO (GA-780684), the ANR Project Loco3D and the ERC LEAP

${ }^{a}$ LAAS-CNRS, 7 Avenue du Colonel Roche, F-31400 Toulouse, France

b Département d'informatique de l'ENS, ENS, CNRS, PSL University, Paris, France

${ }^{c}$ INRIA, Paris, France

*corresponding author: rohan.budhiraja@laas.fr does not rely on any assumptions (like the constant altitude of the Center of Mass (CoM) for the table-cart model). However, to ensure that the two subproblems do not produce divergent and incoherent solutions at the global level, additional constraints are required. These additional constraints are either represented explicitly, for example by using the whole-body kinematics in the centroidal optimization problem [4], or implicitly via proxy constraints to encode the full body behavior [7]. While adding explicit constraints is computationally expensive, kinematic proxy constraints have shown good results [7] [8]. However, it is difficult to define proxy constraints for all the centroidal quantities, especially the Angular Momentum (AM).

An alternative approach introduced in [9] and then exploited in [10], consists of alternating between the reduced and the whole-body problems in a recursive way. In [9], the $\mathrm{CoM}$ and $\mathrm{AM}$ trajectories in the reduced dynamics problem must track the output $\mathrm{CoM}$ and AM trajectories resulting from the whole-body dynamics and vice-versa.

Other approaches have been introduced which compensate for the variations in AM [11], [12]. While they have produced good results, they are not yet able to generate additional momentum based on demands by the whole-body optimizer to enable very dynamic movements, as required (for instance) for fast locomotion [13], where the necessity of angular-momentum (AM) variations is imposed by the motion of the swing leg.

\section{B. Overview of the contribution}

In this paper, we aim to tackle the problem of generating consistent and coherent momentum (CoM and AM) at both centroidal and whole-body levels. We claim that given the efficacy of currently available solvers [14] [15], a feedback from the whole-body dynamics solver towards the centroidal problem would improve the consistency of the global locomotion solution within a few iterations.

However, mathematical rigor should not be avoided in the face of a good heuristic. None of the aforementioned methods ensure good convergence properties of the 2 subproblems to a common and same solution (a consensus), notably for the angular momentum. Rather, they rely on the ability of the individual solvers to produce mutually feasible solutions without properly considering the global structure of the problem. This forces the solvers to have additional robustness in order to account for the lack of structure in the simplified subproblems.

Our main contribution is to introduce a well-posed mathematical formulation that properly enforces the 
consensus between the two subproblems. Rather than giving the solution of the whole-body problem directly to the centroidal optimizer as done by [9], we rely on the Alternating Direction Method of Multipliers (ADMM) technique to handle this feedback communication.

ADMM is an old but well established method for solving convex problems in which the objective is separable into two mutually exclusive cost functions along a set of problem variables. While the method has been around for decades, it was recently reintroduced [16] to solve large scale distributed optimization problems subject to constraints. ADMM provides a feedback to the subproblems in the form of the sum of the residues on the constraints, and in that fashion, it behaves similar to an integral controller. For example, the feedback property has been exploited in [17] to alternate between trajectory and policy optimization.

We find that the robustness and simplicity of this technique makes it an ideal candidate for solving the global optimization problem of locomotion as well. However, a clean splitting of quantities involved in the locomotion problem is required to make the individual subproblems really independent one from each other.

\section{Outline of the paper}

In Section II, we recall the optimal control problem (OCP) dedicated for locomotion and exhibit a complete splitting between centroidal and Lagrangian dynamics. We make obvious the cost implied by solving both subproblems without consensus. In Section III, we detail how ADMM can be exploited to solve the complete OCP by alternatively solving the two subproblems. We gather the details of implementation in Section IV, used to obtained experimental results on the HRP-2 robot in Section V.

\section{The Locomotion Problem}

\section{A. Natural splitting of the robot dynamics}

If we consider a legged robot dotted with $n$ DoF, its whole-body dynamics is represented by the Lagrangian equations of motion:

$$
\left[\begin{array}{l}
\mathbf{H}_{u} \\
\mathbf{H}_{a}
\end{array}\right] \ddot{\mathbf{q}}+\left[\begin{array}{l}
\mathbf{b}_{u} \\
\mathbf{b}_{a}
\end{array}\right]=\left[\begin{array}{l}
\boldsymbol{g}_{u} \\
\boldsymbol{g}_{a}
\end{array}\right]+\left[\begin{array}{c}
\mathbf{0}_{6} \\
\boldsymbol{\tau}
\end{array}\right]+\sum_{k=1}^{K}\left[\begin{array}{l}
\mathbf{J}_{k, u}^{\top} \\
\mathbf{J}_{k, a}^{\top}
\end{array}\right] \boldsymbol{\lambda}_{k}
$$

where $\mathbf{H}$ denotes the joint space inertia matrix, $\mathbf{b}$ encompasses the nonlinear effects, $\boldsymbol{g}$ corresponds to the generalized gravity vector, and $\mathbf{J}_{k}$ is the geometric Jacobian for contact $k$ and $\lambda_{k}$ is the vector of contact forces at contact point $k$. This dynamics can be split into two distinct parts: subscript $u$ denotes the 6 rows correspond to the under-actuated dynamics; subscript $a$ denotes the $n$ rows correspond to the actuated dynamics.

The under-actuated dynamics of (1) is also known as the centroidal dynamics of the robot. It is governed by the Newton-Euler equations of motion which link the variations of the linear momentum and AM to the contact forces:

$$
\begin{aligned}
& m \ddot{\mathbf{c}}=\sum_{k} \boldsymbol{\lambda}_{k}+m \mathbf{g} \\
& \dot{\boldsymbol{L}}=\sum_{k}\left(\boldsymbol{p}_{k}-\mathbf{c}\right) \times \boldsymbol{\lambda}_{k}
\end{aligned}
$$

where $\boldsymbol{p}_{k}$ is the position of the $k^{\text {th }}$ contact point, the operator $\times$ denotes the cross product, $m$ is the total mass of the system, $\mathbf{c}, \dot{\mathbf{c}}, \ddot{\mathbf{c}}$ are the center of mass position, velocity and acceleration vectors and $\boldsymbol{L}, \dot{\boldsymbol{L}}$ are the AM vector and its time derivative.

Thus, a natural splitting appears between two sets of state and control variables, namely:

- the centroidal set called $\mathbf{d}_{c}$ with state $\mathbf{x}_{c}=(\mathbf{c}, \dot{\mathbf{c}}, \boldsymbol{L})$ and control $\mathbf{u}_{c}=\left(\boldsymbol{\lambda}_{1}, \boldsymbol{\lambda}_{2}, \ldots, \boldsymbol{\lambda}_{k}\right)$;

- the Lagrangian set named $\mathbf{d}_{l}$ with state $\mathbf{x}_{l}=(\mathbf{q}, \dot{\mathbf{q}})$ and control $\mathbf{u}_{l}=\tau$.

\section{B. The global Optimal Control Problem for locomotion}

Consider the scenario where the set of contact phases $\mathbf{S}$ and their corresponding contact timings $\Delta t_{s}$ are already defined. Further, if we assume that the actuators are capable enough to provide sufficient torque (which is true for current generation of robots), it is possible to split the global motion planning Optimal Control Problem (OCP) between two states of the robot $\left(\boldsymbol{x}_{\text {init }}, \boldsymbol{x}_{\text {final }}\right)$ into two hierarchical stages, which successively solve for $\underline{\mathbf{d}}_{c}$ and $\underline{\mathbf{d}}_{l}$. However, such an approach mandates that the solution of the first OCP is feasible for the second OCP. The kinematic feasibility condition between $\mathbf{q}$ and $\mathbf{c}$ has been explored [7] previously by our team.

The motion planning OCP, governed by the dynamics defined by (1) and (2), and the feasibility criteria given in [7] is given by ${ }^{1}$ :

$$
\begin{array}{cl}
\underset{\underline{\mathbf{d}}_{c}, \underline{\mathbf{d}}_{l}}{\operatorname{minimize}} & \sum_{s=1}^{S} \int_{t_{s}}^{t_{s}+\Delta t_{s}} \ell_{s}^{c}\left(\mathbf{d}_{c}\right) d t+\sum_{s=1}^{S} \int_{t_{s}}^{t_{s}+\Delta t_{s}} \ell_{s}^{l}\left(\mathbf{d}_{l}\right) d t \\
\text { subject to } & \forall t \quad \mathbf{c}=\operatorname{CoM}(\mathbf{q}) \\
& \forall t \quad\left[\begin{array}{c}
m \dot{\mathbf{c}} \\
\boldsymbol{L}
\end{array}\right]=\boldsymbol{A}_{g}(\mathbf{q}) \dot{\mathbf{q}} \\
\forall t & \boldsymbol{\lambda}=g_{\lambda}(\mathbf{q}, \dot{\mathbf{q}}, \boldsymbol{\tau}) \\
\forall t & \boldsymbol{\lambda} \in \mathcal{K} \\
\forall t & g_{\lambda}(\mathbf{q}, \dot{\mathbf{q}}, \boldsymbol{\tau}) \in \mathcal{K} \\
\forall t & \dot{\mathbf{x}}_{c}=f_{c}\left(\mathbf{d}_{c}\right) \\
\forall t & \dot{\mathbf{x}}_{l}=f_{l}\left(\mathbf{d}_{l}\right) \\
& \mathbf{x}_{c}(0) \text { is given, } \mathbf{x}_{c}(T) \text { is viable } \\
& \mathbf{x}_{l}(0) \text { is given, } \mathbf{x}_{l}(T) \text { is viable }
\end{array}
$$

where $s$ is the index of the contact phase, $t_{s}$ is the start time of the contact phase $s . \ell_{s}^{c}$ and $\ell_{s}^{l}$ are local cost functions related to the phase. $\mathcal{K}$ denotes the admissible set of the friction forces corresponding to zero slippage. $\boldsymbol{A}_{g}$ is the so-called centroidal momentum matrix [3], and CoM maps the current joint configuration $\mathbf{q}$ to the center of mass position. $g_{\lambda}$ is the mapping between the whole-body dynamics and contact forces, and may be dependent [15] or independent [18] from $\tau$, depending on the choice of contact model.

\footnotetext{
${ }^{1}$ Note that for all variables, underlines denote a trajectory of the variable over time. Similarly he dependency to the time variable is kept implicit i.e. $\forall t \mathbf{c}$ is preferred to $\forall t \mathbf{c}(t)$.
} 
Most of constraints and the two cost terms only depend on one of the two groups of variables $\underline{\mathbf{d}}_{c}, \underline{\mathbf{d}}_{l}: \ell_{s}^{c}\left(\mathbf{d}_{c}\right)$, (3e), (3g) and (3i) define a problem over the centroidal dynamics ; $\ell_{s}^{c}\left(\mathbf{d}_{l}\right),(3 \mathrm{f}),(3 \mathrm{~h})$ and $(3 \mathrm{j})$ define a problem over the Lagrangian dynamics. The two groups are coupled by constraints (3b), (3c) and (3d). One way to solve the two problems independently is to replace these three coupling constraints by some proxy constraint, i.e. reformulation which enforces the existence of a global consensus solution acceptable by both subproblems. In [7], we have proposed to learn such a proxy constraints for the centroidal optimization. In the experiments of this paper, we will use again this learned proxy in the initial step of our algorithms.

Constraints (3e) and (3f) are redundant (i.e. (3e) and (3d) implies (3f)). Both of these constraints impose non-slippage conditions on the contact forces. (3e) and (3g) enforce consistent centroidal dynamics (2), while (3f) and (3h) enforce consistence of the Lagrangian dynamics (1) with respect to the contact model. We explicitly formulate both constraints to make the split evident. Similar remark holds for initial and terminal conditions (3i) and (3j). As terminal constraints are often difficult to formulate in practice, they should likely be replaced by stopping motion conditions (e.g. capturability) [19].

The near-perfect split has already been observed [12]. In this nice work, the observation was mostly used to justify the classical approach of separately solving each subproblem. Here we rather want to insist on the coupling and pave the way to the use of alternated descent.

\section{Why should we alternate?}

In a first implementation, it is often proposed to first compute the centroidal pattern and then track it by solving the Lagrangian dynamics. The whole-body movement is usually computed with an Inverse Kinematics (IK)/Inverse Dynamics (ID), which are theoretically equivalent to solving the Lagrangian part of (3) but with a void horizon $T=0$. With such a simplification, it is desired that the AM output from the centroidal problem must match near perfectly to the $A M$ requirement by the Lagrangian part. However, this is not possible without some form of feedback (in the form of alternation) on the AM value. While this requirement is known by many teams, we believe that it is not sufficiently documented and explain why alternating is important.

Momentum variations are caused by the forces exerted by the environment at the contact level, and they result in the motion of the limbs (also called "gesticulation" [13]). As the centroidal model does not understand gesticulation(which depends on the kinematic and dynamic structure of the robot), it is not possible to get the correct momentum estimate when considering only the centroidal quantities. Consider the example of the biped locomotion gait: an astronaut mimicking walk in deep space would rotate (pitch rotation) on the spot (same for a falling cat [13]). This is due to the asymmetry in the movements of the limbs during the forward and backward motions. As we are not rotating during locomotion, we can conclude that we exert some contact forces to counterbalance this rotation effect. These extra forces, which are required due to gesticulation, cannot be decided from the centroidal model alone. Consequently, trying to approximately match the AM computed by the centroidal solver is a bad idea. The same is also true for CoM (linear momentum) trajectory, which should change to account for the change in forces, according to (2).

A pragmatic solution is to compute the centroidal pattern by trying to match the AM that the limbs will generate. This implicitly suggests that we are not expecting to use the $\mathrm{AM}$ variations to improve the walk, but we are just trying to compensate for it. This is the standard implementation of the table-cart pattern generator, by adding a second stage of ZMP-CoM computation [11]. It has also been proposed to couple an IK with a centroidal solver [5]. In both cases, it has been experimentally observed that alternating twice is enough to obtain a consensus. However, no theoretical basis has yet been provided. In Sec III, we propose to alternate using an existing theoretical framework which forces consensus as an output of the optimization.

\section{Alternating Method FOR LOCOMOTION}

In this section, we first review the ADMM technique. We then apply it on the global OCP for locomotion (3).

\section{A. Alternating Direction Method of Multipliers}

ADMM is a simple optimization technique to solve constrained problems of the form:

$$
\begin{array}{ll}
\underset{\boldsymbol{x}, \boldsymbol{z}}{\operatorname{minimize}} & l_{1}(\boldsymbol{x})+l_{2}(\boldsymbol{z}) \\
\text { subject to } & A \boldsymbol{x}+B \boldsymbol{z}=\boldsymbol{c}
\end{array}
$$

where the cost function is composed of two separable objectives $l_{1}(\boldsymbol{x})$ and $l_{2}(\boldsymbol{z})$. The main idea behind ADMM is to exploit this splitting between cost terms in a recursive manner, allowing to solve simpler problems than the original one [16]. This precise point can be highlighted by writing the augmented Lagrangian associated with the constrained optimization problem (4):

$$
\begin{aligned}
\mathcal{L}_{\rho}(\boldsymbol{x}, \boldsymbol{z}, \boldsymbol{y})=l_{1}(\boldsymbol{x})+l_{2}(\boldsymbol{z}) & +\boldsymbol{y}^{T}(A \boldsymbol{x}+B \boldsymbol{z}-\boldsymbol{c}) \\
& +\frac{\rho}{2}\|A \boldsymbol{x}+B \boldsymbol{z}-\boldsymbol{c}\|_{2}^{2}
\end{aligned}
$$

where $\boldsymbol{y}$ is the vector of dual variables associated with the constraint $A \boldsymbol{x}+B \boldsymbol{z}=\boldsymbol{c}$ and $\rho>0$ is the penalty parameter which penalizes the violations of this constraint. The solution is then found by the following steps recursions ${ }^{2}$ :

$$
\begin{aligned}
& \boldsymbol{x}^{k+1}=\underset{\boldsymbol{x}}{\operatorname{argmin}} \mathcal{L}_{\rho}\left(\boldsymbol{x}, \boldsymbol{z}^{k}, \boldsymbol{y}^{k}\right) \\
& \boldsymbol{z}^{k+1}=\underset{\boldsymbol{z}}{\operatorname{argmin}} \mathcal{L}_{\rho}\left(\boldsymbol{x}^{k+1}, \boldsymbol{z}, \boldsymbol{y}^{k}\right) \\
& \boldsymbol{y}^{k+1}=\boldsymbol{y}^{k}+\rho(A \boldsymbol{x}+B \boldsymbol{z}-\boldsymbol{c})
\end{aligned}
$$

Problem (6a) and (6b) are minimization over $l_{1}$ and $l_{2}$ respectively (with an additional quadratic term). It is also worth noticing that the dual variable $\boldsymbol{y}$, through the

\footnotetext{
${ }^{2}$ Throughout the paper, superscripts are used to refer to the current iteration of the solver
} 
update (6c) acts as an integral term by collecting the residues on the consensus between the two subproblems, and forces the residual to converge to 0 along the iterations.

\section{B. ADMM for locomotion rational}

ADMM provides a way for us to exploit the splitting of dynamic variables exposed in Sec II-B, and defines a mathematical framework to feedback and optimize the AM variable inside the centroidal OCP.

OCP (3) does not match exactly the pattern of (4): (3) has three nonlinear coupling constraints((3b), (3c), (3d)) and additional decoupled constraints( $(3 \mathrm{e})$ to $(3 \mathrm{j}))$. For the 3 semi-infinite (i.e. defined $\forall t$ ) coupling constraints, we have to introduce 3 multipliers functions of time. For the additional decoupled constraints, we handle them in the solvers of each subproblems. Let us remember that the partial solutions $\underline{\mathbf{d}}_{c}$ and $\underline{\mathbf{d}}_{l}$ of each subproblems should respect these additional constraints. In this section, we explain the alternating algorithm with the hypothesis that some oracles can be called to provide the optimum of the two subproblems. The next section will describe with more details which centroidal and whole-body solvers we used for the experiments.

Non-linearity is a theoretical issue and it makes the problem non-convex. Convergence guarantee with ADMM are yet only obtained for convex problems with linear constraints. Yet ADMM with non-convex problems would act as just another local optimizer; and we at least know that the linearization of (3) will converge. In practice, ADMM is often used, with good empirical results, for solving problems with non-convex objectives [16] [20], and for non-linear constraints [21].

\section{Application of ADMM to the locomotion problem}

Let us associate with each coupling constraint a residual function:

$$
\begin{array}{ll}
\forall t & \boldsymbol{r}_{c}(\mathbf{c}, \mathbf{q})=\mathbf{c}-\operatorname{CoM}(\mathbf{q}) \\
\forall t & \boldsymbol{r}_{m}(\dot{\mathbf{c}}, \boldsymbol{L}, \mathbf{q}, \dot{\mathbf{q}})=\left[\begin{array}{c}
m \dot{\mathbf{c}} \\
\boldsymbol{L}
\end{array}\right]-\boldsymbol{A}_{g}(\mathbf{q}) \dot{\mathbf{q}} \\
\forall t & \boldsymbol{r}_{\lambda}(\lambda, \mathbf{q}, \dot{\mathbf{q}}, \tau)=\lambda-g_{\lambda}(\mathbf{q}, \dot{\mathbf{q}}, \tau)
\end{array}
$$

Residuals $\underline{\boldsymbol{r}}_{c}, \underline{\boldsymbol{r}}_{m}, \underline{\boldsymbol{r}}_{g}$ respectively corresponds to constraints (3b), (3c), (3d). We also respectively define $\underline{\boldsymbol{y}}_{c}, \underline{\boldsymbol{y}}_{m}, \underline{\boldsymbol{y}}_{g}$ as the multipliers corresponding to these 3 constraints.

For convenience, let us define $\underline{r}$ as the augmentation of (3) (i.e. sum of linear and quadratic penalization):

$$
\underline{\boldsymbol{r}}\left(\underline{\mathbf{d}}_{c}, \underline{\mathbf{d}}_{l}, \underline{\boldsymbol{y}}\right)=\sum_{k=m, c, \lambda} \int_{0}^{T_{f}} \boldsymbol{y}_{k}^{T}(t) \boldsymbol{r}_{k}(t)+\frac{\rho_{k}(t)}{2}\left\|\boldsymbol{r}_{k}(t)\right\|_{2}^{2} d t
$$

We can now separate the augmented Lagrangian of the global
OCP into centroidal and full body parts:

$$
\begin{aligned}
& \mathcal{L}_{\rho}^{c}\left(\underline{\mathbf{d}}_{c}, \underline{\mathbf{d}}_{l}, \underline{\boldsymbol{y}}\right)=\sum_{s=1}^{S} \int_{t_{s}}^{t_{s}+\Delta t_{s}} \ell_{s}^{c}\left(\mathbf{d}_{c}\right) d t+\underline{\boldsymbol{r}} \\
& \mathcal{L}_{\rho}^{l}\left(\underline{\mathbf{d}}_{c}, \underline{\mathbf{d}}_{l}, \underline{\boldsymbol{y}}\right)=\sum_{s=1}^{S} \int_{t_{s}}^{t_{s}+\Delta t_{s}} \ell_{s}^{l}\left(\mathbf{d}_{l}\right) d t+\underline{\boldsymbol{r}}
\end{aligned}
$$

where $y$ is the stack of the 3 multipliers. Note that the multipliers are trajectories of vectors, while $\rho_{c}, \rho_{m}, \rho_{\lambda}$ are trajectories of scalars.

Using the definition of ADMM from Sec III-A, the global OCP can thus be solved by the following iterations:

$$
\begin{aligned}
& \underline{\mathbf{d}}_{c}^{k+1}=\underset{\underline{\mathbf{d}}_{c}}{\operatorname{argmin}} \mathcal{L}_{\rho}^{c}\left(\underline{\mathbf{d}}_{c}, \underline{\mathbf{d}}_{l}^{k}, \underline{\boldsymbol{y}}^{k}\right) \text { subject to }(3 e),(3 g),(3 i) \\
& \underline{\mathbf{d}}_{l}^{k+1}=\underset{\underline{\mathbf{m}}^{2}}{\operatorname{argmin}} \mathcal{L}_{\rho}^{l}\left(\underline{\mathbf{d}}_{c}^{k+1}, \underline{\mathbf{d}}_{l}, \underline{\boldsymbol{y}}^{k}\right) \text { subject to }(3 f),(3 h),(3 j) \\
& \forall t \quad \boldsymbol{y}_{c}^{k+1}=\boldsymbol{y}_{c}^{k}+\rho_{c}\left(\mathbf{c}^{k+1}-C o M\left(\mathbf{q}^{k+1}\right)\right) \\
& \forall t \quad \boldsymbol{y}_{m}^{k+1}=\boldsymbol{y}_{m}^{k}+\rho_{m}\left(\left[\begin{array}{c}
m \dot{\mathbf{c}}^{k+1} \\
\boldsymbol{L}^{k+1}
\end{array}\right]-\boldsymbol{A}_{g}\left(\mathbf{q}^{k+1}\right) \dot{\mathbf{q}}^{k+1}\right) \\
& \forall t \quad \boldsymbol{y}_{\lambda}^{k+1}=\boldsymbol{y}_{\lambda}^{k}+\rho_{\lambda}\left(\lambda^{k+1}-g_{\lambda}\left(\mathbf{q}^{k+1}, \dot{\mathbf{q}}^{k+1}, \tau^{k+1}\right)\right)
\end{aligned}
$$

\section{Alternating Using Dedicated solvers}

In this section, we describe the practical details of our implementation, enabling us to simply add the alternating descent on top of our existing frameworks for solving centroidal and whole-body dynamics OCP, with only a minor additional cost in terms of development.

\section{A. The locomotion pipeline}

Our locomotion pipeline called Loco3d is described in detail in [22]. It is composed of three main stages:

1) The Contact Sequence Generator: It is a randomized motion planner [23], which ensures the feasibility of the sequence at the kinematic level, with real-time performances;

2) The Centroidal Optimizer: It solves the trajectory optimization problem over the centroidal dynamics using feasibility measures to enforce the kinematic feasibility of the solution with respect to the whole-body problems. We use MUSCOD-II [24], which implements an efficient multiple-shooting algorithm particularly suited for the multiple contact phases of the locomotion problem. The output of the solver is the centroidal trajectories $\underline{\mathbf{d}}_{c}$;

3) The Whole-Body Optimizer: It solves the full-body trajectory optimization problem subject to the dynamics constraints. We use a new iterative Differential Dynamic Programming solver [15] which accounts for the contact constraints in the dynamics of the problem. The output of the solver is the whole-body trajectories $\underline{\mathbf{d}}_{l}$.

\section{B. On the advantages of scaling the dual variables}

Scaling the dual variables through $z=\frac{y}{\rho}$ is more convenient to implement as it combines under a single norm minimization both the linear and quadratic terms in 
the augmented Lagrangian (9). The subproblems are then reduced to simply minimize the residual squared norm of the constraints linking the two problems [16].

\section{The ADMM solver for locomotion}

By using $\underline{\boldsymbol{z}}$, we can simplify the notation further by collecting the constraints (3b), (3c) and (3d) into a single quantity. For that aim, let us define $\Phi_{c}$ to contain the elements $(\boldsymbol{c}, m \dot{\mathbf{c}}, \boldsymbol{L}$ and $\boldsymbol{\lambda})$. Similarly, let us define $\Phi_{l}$ to contain the mappings $\left(\operatorname{CoM}(\mathbf{q}), \boldsymbol{A}_{g}(\mathbf{q}) \dot{\mathbf{q}}\right.$ and $\left.g_{\lambda}(\mathbf{q}, \dot{\mathbf{q}}, \boldsymbol{\tau})\right)$. Let us define addition/subtraction operations on $\Phi$ to be the addition/subtraction on its corresponding elements and a function DMap which maps the centroidal $\left(\mathbf{d}_{c}\right)$ and whole-body $\left(\mathbf{d}_{l}\right)$ variables onto these elements. The iterations (10) can be then written equivalently as Algorithm 1. In Algorithm 1, the first input to the solvers is the solution of the previous iteration (as a warm start), and the second input is the reference to be tracked for the augmented Lagrangian quadratic costs.

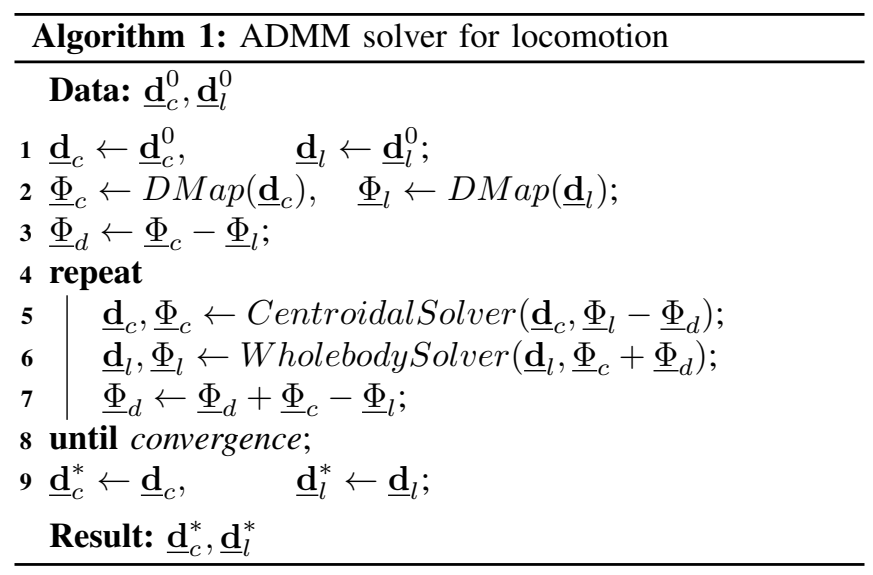

\section{Initializing the dual variables}

Many of the currently available centroidal and whole-body dynamics solvers are tailored for producing solutions which are almost always mutually feasible [12] [15] [25]. As a result,with this assumption of feasibility, a simple feedback from the whole-body solver to the centroidal solver generates acceptable result within the second iteration [12]. On the other hand, since ADMM transmits the residual of the two subproblems and not the output, an uninitialized ADMM solver would overshoot and only promise convergence from the third iteration, as we later see in Sec V. There is indeed a minimum number of iterations to synchronize the two subproblems.

However, this extra iteration of the solver could be avoided by setting the dual after the first iteration as $\underline{\Phi}_{d}^{1} \leftarrow \underline{\Phi}_{c}^{1}$. This change makes the feedback from the first iteration to be equal to the output, and the knowledge of individual solvers can then be exploited to converge within two iterations. Indeed, the second iteration of the ADMM solver then becomes equivalent to [12], and would provide similar results without affecting convergence. While not used in this paper, this trick needs to be evaluated further.

\section{E. Key observations for the centroidal solver}

In the centroidal OCP, there is a redundancy in the state. The state $\mathbf{c}, \dot{\mathbf{c}}, \boldsymbol{L}$ has a dimension of 9 , while the control can be represented in a dimension 6 (with a centroidal wrench acting on the center of mass). This redundancy is usually suppressed by predefining or regularizing $\boldsymbol{L}$ [7], and solving for the rest. The same principle can be exploited in the present case, by predefining $\underline{\boldsymbol{L}}^{k+1}$ to be equal to the AM part of $\underline{\Phi}_{l}^{k}-\underline{\Phi}_{d}^{k}$ and solving the centroidal step only for $\underline{\mathbf{c}}^{k+1}, \underline{\dot{\mathbf{c}}}^{k+1}, \underline{\boldsymbol{\lambda}}^{k+1}$. This method ensures that the AM is always consistent with the full body dynamics.

As a result, angular part of $\boldsymbol{r}_{m}$ is always zero after the centroidal step of (10). Equivalently, variations in the AM part of $\underline{\Phi}_{d}^{k+1}$ are only dependent on the variations in the AM part of $\underline{\Phi}_{l}^{k+1}$ ( $\boldsymbol{L}$ is given as a feedback from the full-body OCP to centroidal OCP via $\boldsymbol{r}_{m}$ )

\section{F. Key observations for whole-body solver}

During the full-body step of (10), the knowledge of $\lambda^{k+1}$ from the centroidal step can be exploited to ensure that the Constraint (3f) is never violated. In the full-body step (by the mapping $\left.g_{\lambda}\right) \boldsymbol{\lambda}$ is dependent on $\mathbf{q}, \dot{\mathbf{q}}, \boldsymbol{\tau}$. A predefined $\boldsymbol{\lambda}_{r e f}^{k+1}$ provides a good reference for the contact forces. With a good tracking, Constraint (3f) can be relaxed. An implementation of the full-body step with rigid contacts which exploits such a reference tracking is proposed in [15]. Note that in [15] c and $\lambda$ were being tracked in the full-body OCP, while in the present formulation they are updated during the dual ascent.

\section{EXPERIMENTAL RESULTS}

In this section, we validate and highlight the efficiency of the proposed approach in simulation. For that purpose, we study the convergence properties of our solver on a simplified version of the humanoid robot HRP-2 only composed of its lower limbs. We generate a walking motion composed of 3 steps of $40 \mathrm{~cm}$ step length, depicted in Fig. 1.

\section{A. Cost functions}

1) The centroidal cost function: for the first iteration of the centroidal solver, we minimize the log barrier on the feasibility measure of the CoM [7], and we regularize the CoM velocity (weight: 10) and contact forces (weight: $10^{-4}$ for linear and $10^{-2}$ for angular) with quadratic costs. For subsequent iterations, we solely minimize the dual terms with $\rho_{c}=1.0, \rho_{m}=10^{-2}$ and $\rho_{\lambda}=10^{-4}$.

2) The whole-body cost function: For the whole-body solver, we use only quadratic costs to regularize the posture (weight: $10^{-6}$ ) and the free-flyer orientation (weight: 20) in addition to the augmented Lagrangian terms.

\section{B. Convergence analysis}

We stopped the alternating resolution after only 10 iterations of the solver described in Algorithm 1. While 3 iterations were empirically sufficient to compute a feasible motion in simulation, we show here the continuing convergence. As shown in Fig. 2, the total residuals of the matching constraints decrease rapidly over the first three 

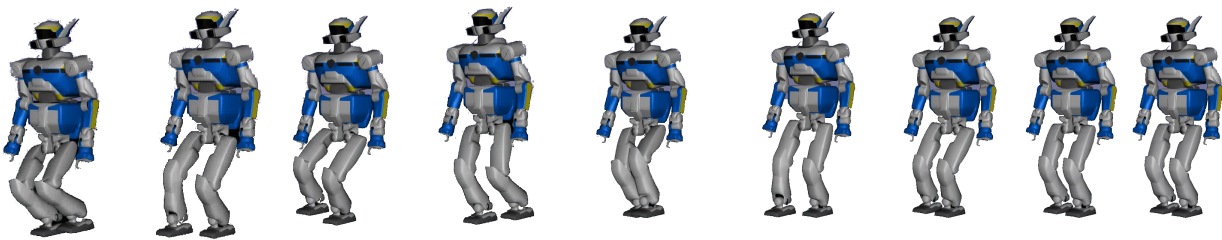

Fig. 1: Walking sequence generated for HRP-2 robot using the proposed ADMM solver.

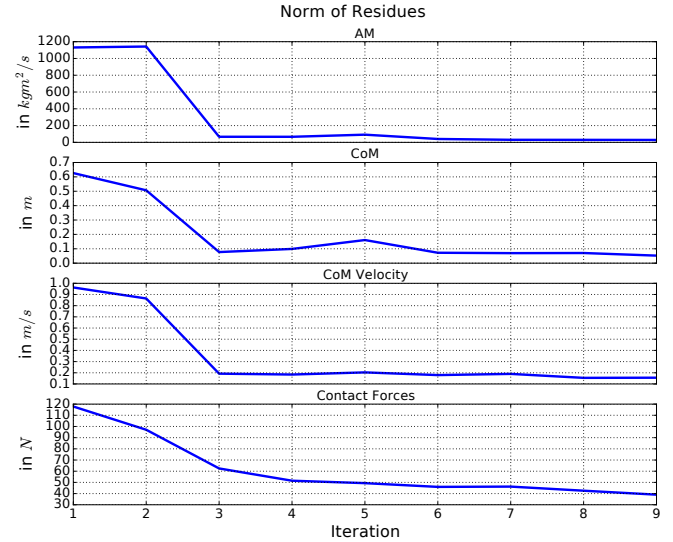

Fig. 2: Evolution of the total norm of the constraint residual along the iterations of the ADMM solver.

iterations and more slowly after. This really means that the two problem are able to find a consensus in very few iterations. This behavior is also well depicted in Fig. 3, where one can observe the CoM trajectories of both centroidal and whole-body subproblems. Already at iteration 3, the two trajectories match almost perfectly.

The mismatching of CoM and AM quantities is also reflected in Fig. 4. The two first iterations show a large mismatch between the centroidal and the whole-body problems, but the residual decreases rapidly towards the value 0 . This phenomena can be explained by the delay induced by the ADMM approach: the solver overshoots and then builds and maintains a consensus between the two subproblems as discussed in Sec IV-D.

\section{CONClusion ANd Future Work}

In this paper, we have introduced a systematic approach to build a consensus on the dynamics constraints between the centroidal and whole-body optimization problems. Based on previous observation between the nice articulation between under-actuated and actuated dynamics of legged robots, we have given a mathematical framework to separate the two problems, and proposed a solution which iterates between the two subproblems and maintains consensus in the solutions. Finally, we demonstrate with a walking sequence on HRP-2 the performance of the solver.

While some heuristics are available allowing similar behaviors, the ADMM solver encompasses the dynamics constraints within the framework of the solver itself. In this way, the current method deals with not only individual and separated subproblems, but tackles the global problem

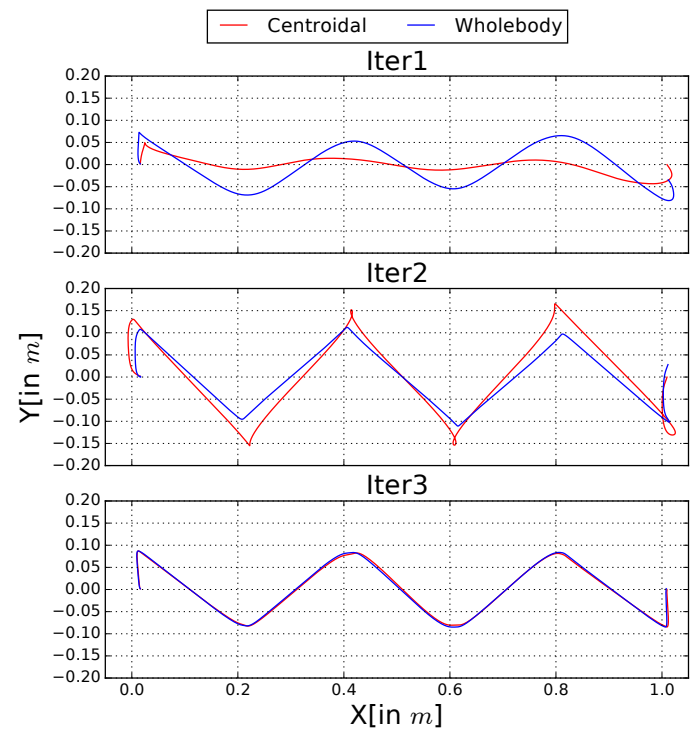

Fig. 3: CoM trajectory in the $\mathrm{XY}$ plane for the first three iterations of the ADMM solver.

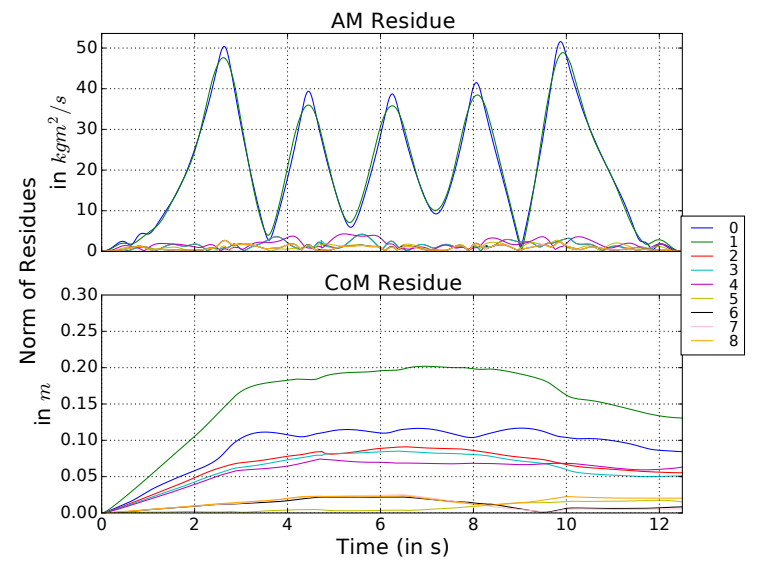

Fig. 4: Residue between the centroidal and whole-body trajectory of $\mathrm{AM}$ and CoM over the iterations.

defined in (3). To the best of our knowledge, this is the first time that a consensus between centroidal and Lagrangian dynamic solvers is obtained based on theoretical grounding.

However, even if this solver currently solves the full-body dynamics problem, it assumes a dependence on the upstream contact planner to provide feasible contact planning. Thus, we need to ensure a similar consistency between the upstream contact planner and the full body optimizer as well. This is a future avenue of research for the community. 


\section{REFERENCES}

[1] S. Kajita, F. Kanehiro, K. Kaneko, K. Yokoi, and H. Hirukawa, "The 3D linear inverted pendulum mode: a simple modeling for a biped walking pattern generation," in IEEE/RSJ International Conference on Intelligent Robots and Systems, vol. 1. IEEE, 2001, pp. 239-246. [Online]. Available: http://ieeexplore.ieee.org/document/973365/

[2] J. Pratt, J. Carff, S. Drakunov, and A. Goswami, "Capture Point: A Step toward Humanoid Push Recovery," in 2006 6th IEEE-RAS International Conference on Humanoid Robots. IEEE, dec 2006, pp. 200-207. [Online]. Available: http://ieeexplore.ieee.org/document/ $4115602 /$

[3] D. E. Orin, A. Goswami, and S.-H. Lee, "Centroidal dynamics of a humanoid robot," Autonomous Robots, vol. 35, no. 2-3, pp. 161-176, oct 2013. [Online]. Available: http://link.springer.com/10. 1007/s10514-013-9341-4

[4] H. Dai, A. Valenzuela, and R. Tedrake, "Whole-body motion planning with centroidal dynamics and full kinematics," in 2014 IEEE-RAS International Conference on Humanoid Robots. IEEE, nov 2014, pp. 295-302. [Online]. Available: http://ieeexplore.ieee.org/document/ 7041375/

[5] A. Herzog, N. Rotella, S. Schaal, and L. Righetti, "Trajectory generation for multi-contact momentum control," in 2015 IEEE-RAS 15th International Conference on Humanoid Robots (Humanoids). IEEE, nov 2015, pp. 874-880. [Online]. Available: http://ieeexplore. ieee.org/document/7363464/

[6] J. Carpentier, S. Tonneau, M. Naveau, O. Stasse, and N. Mansard, "A versatile and efficient pattern generator for generalized legged locomotion," in 2016 IEEE International Conference on Robotics and Automation (ICRA). IEEE, may 2016, pp. 3555-3561. [Online]. Available: http://ieeexplore.ieee.org/document/7487538/

[7] J. Carpentier, R. Budhiraja, and N. Mansard, "Learning Feasibility Constraints for Multicontact Locomotion of Legged Robots," in Robotics: Science and Systems XIII. Robotics: Science and Systems Foundation, jul 2017. [Online]. Available: http://www. roboticsproceedings.org/rss13/p31.pdf

[8] S. Tonneau, P. Fernbach, A. Del Prete, J. Pettré, and N. Mansard, "2PAC: Two-Point Attractors for Center Of Mass Trajectories in Multi-Contact Scenarios," ACM Transactions on Graphics, vol. 37, no. 5, pp. 1-14, oct 2018. [Online]. Available: http: //dl.acm.org/citation.cfm?doid=3278329.3213773

[9] A. Herzog, S. Schaal, and L. Righetti, "Structured contact force optimization for kino-dynamic motion generation," in 2016 IEEE/RSJ International Conference on Intelligent Robots and Systems (IROS). IEEE, oct 2016, pp. 2703-2710. [Online]. Available: http://ieeexplore.ieee.org/document/7759420/

[10] B. Ponton, A. Herzog, S. Schaal, and L. Righetti, "A convex model of humanoid momentum dynamics for multi-contact motion generation," in 2016 IEEE-RAS 16th International Conference on Humanoid Robots (Humanoids). IEEE, nov 2016, pp. 842-849. [Online]. Available: http://ieeexplore.ieee.org/document/7803371/

[11] S. Kajita, F. Kanehiro, K. Kaneko, K. Fujiwara, K. Harada, K. Yokoi, and $\mathrm{H}$. Hirukawa, "Biped walking pattern generation by using preview control of zero-moment point," in IEEE International Conference on Robotics and Automation. IEEE, 2003, pp. 1620-1626. [Online]. Available: http://ieeexplore.ieee.org/document/1241826/

[12] A. Herzog, N. Rotella, S. Mason, F. Grimminger, S. Schaal, and L. Righetti, "Momentum control with hierarchical inverse dynamics on a torque-controlled humanoid," Autonomous Robots, vol. 40, no. 3, pp. 473-491, mar 2016. [Online]. Available: http://link.springer.com/10.1007/s10514-015-9476-6

[13] P.-B. Wieber, "Holonomy and nonholonomy in the dynamics of articulated motion," in Lecture Notes in Control and Information
Sciences, vol. 340. Berlin, Heidelberg: Springer Berlin Heidelberg, 2006, pp. 411-425. [Online]. Available: http://link.springer.com/10. 1007/978-3-540-36119-0\{_\}20

[14] J. Carpentier and N. Mansard, "Multicontact Locomotion of Legged Robots," IEEE Transactions on Robotics, vol. 34, no. 6, pp. 1441-1460, dec 2018. [Online]. Available: https://ieexplore.ieee.org/ document/8558661/https://hal.archives-ouvertes.fr/hal-01520248/

[15] R. Budhiraja, J. Carpentier, C. Mastalli, and N. Mansard, "Differential Dynamic Programming for Multi-Phase Rigid Contact Dynamics," in 2018 IEEE-RAS 18th International Conference on Humanoid Robots (Humanoids). IEEE, nov 2018, pp. 1-9. [Online]. Available: https://ieeexplore.ieee.org/document/8624925/

[16] S. Boyd, N. Parikh, E. Chu, B. Peleato, and J. Eckstein, "Distributed Optimization and Statistical Learning via the Alternating Direction

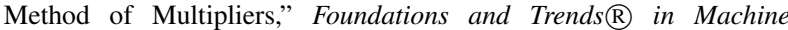
Learning, vol. 3, no. 1, pp. 1-122, 2010. [Online]. Available: http://www.nowpublishers.com/article/Details/MAL-016

[17] I. Mordatch and E. Todorov, "Combining the benefits of function approximation and trajectory optimization," in Robotics: Science and Systems X. Robotics: Science and Systems Foundation, jul 2014. [Online]. Available: http://www.roboticsproceedings.org/rss10/p52.pdf

[18] M. Neunert, M. Stauble, M. Giftthaler, C. D. Bellicoso, J. Carius, C. Gehring, M. Hutter, and J. Buchli, "Whole-Body Nonlinear Model Predictive Control Through Contacts for Quadrupeds," IEEE Robotics and Automation Letters, vol. 3, no. 3, pp. 1458-1465, jul 2018 [Online]. Available: http://ieeexplore.ieee.org/document/8276298/

[19] P.-B. Wieber, "Viability and predictive control for safe locomotion," in 2008 IEEE/RSJ International Conference on Intelligent Robots and Systems. IEEE, sep 2008, pp. 1103-1108. [Online]. Available: http://ieeexplore.ieee.org/document/4651022/

[20] Y. Wang, W. Yin, and J. Zeng, "Global Convergence of ADMM in Nonconvex Nonsmooth Optimization," Journal of Scientific Computing, vol. 78, no. 1, pp. 29-63, jan 2019. [Online]. Available: http://link.springer.com/10.1007/s10915-018-0757-z

[21] M. Benning, F. Knoll, C.-B. Schönlieb, and T. Valkonen, "Preconditioned ADMM with Nonlinear Operator Constraint," IFIP Conference on System Modeling and Optimization, vol. 494, pp. 117-126, 2016. [Online]. Available: http: //link.springer.com/10.1007/978-3-319-55795-3\{_\}10

[22] J. Carpentier, A. D. Prete, S. Tonneau, T. Flayols, F. Forget, A. Mifsud, K. Giraud, D. Atchuthan, P. Fernbach, R. Budhiraja, M. Geisert, J. Solà, O. Stasse, and N. Mansard, "Multi-contact Locomotion of Legged Robots in Complex Environments - The Loco3D project," in RSS Workshop on Challenges in Dynamic Legged Locomotion, Boston, United States, jul 2017, p. 3p. [Online]. Available: https://hal.laas.fr/hal-01543060

[23] S. Tonneau, N. Mansard, C. Park, D. Manocha, F. Multon, and J. Pettré, "A Reachability-Based Planner for Sequences of Acyclic Contacts in Cluttered Environments.” Springer, Cham, 2018, pp. 287-303. [Online]. Available: http://link.springer.com/10.1007/ 978-3-319-60916-4\{-\}17

[24] D. B. Leineweber, I. Bauer, H. G. Bock, and J. P. Schlöder, "An efficient multiple shooting based reduced SQP strategy for large-scale dynamic process optimization. Part 1: theoretical aspects," Computers \& Chemical Engineering, vol. 27, no. 2, pp. 157-166, feb 2003. [Online]. Available: https://www.sciencedirect.com/science/article/pii/ S0098135402001588

[25] J. Carpentier and N. Mansard, "Analytical Derivatives of Rigid Body Dynamics Algorithms," in Robotics: Science and Systems XIV. Robotics: Science and Systems Foundation, jun 2018. [Online]. Available: http://www.roboticsproceedings.org/rss14/p38.pdf 